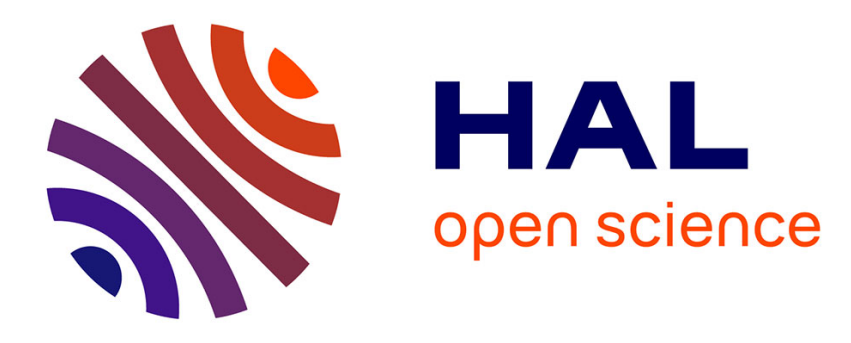

\title{
Costly dispersal can destabilize the homogeneous equilibrium of a metapopulation
}

\author{
E. Éva Kisdi
}

\section{To cite this version:}

E. Éva Kisdi. Costly dispersal can destabilize the homogeneous equilibrium of a metapopulation. Journal of Theoretical Biology, 2009, 262 (2), pp.279. 10.1016/j.jtbi.2009.09.032 * hal-00554650

\author{
HAL Id: hal-00554650 \\ https://hal.science/hal-00554650
}

Submitted on 11 Jan 2011

HAL is a multi-disciplinary open access archive for the deposit and dissemination of scientific research documents, whether they are published or not. The documents may come from teaching and research institutions in France or abroad, or from public or private research centers.
L'archive ouverte pluridisciplinaire HAL, est destinée au dépôt et à la diffusion de documents scientifiques de niveau recherche, publiés ou non, émanant des établissements d'enseignement et de recherche français ou étrangers, des laboratoires publics ou privés. 


\section{Author's Accepted Manuscript}

Costly dispersal can destabilize the homogeneous equilibrium of a metapopulation

Éva Kisdi

PII:

S0022-5193(09)00470-6

DOI: doi:10.1016/j.jtbi.2009.09.032

Reference: YJTBI 5723

To appear in: $\quad$ Journal of Theoretical Biology

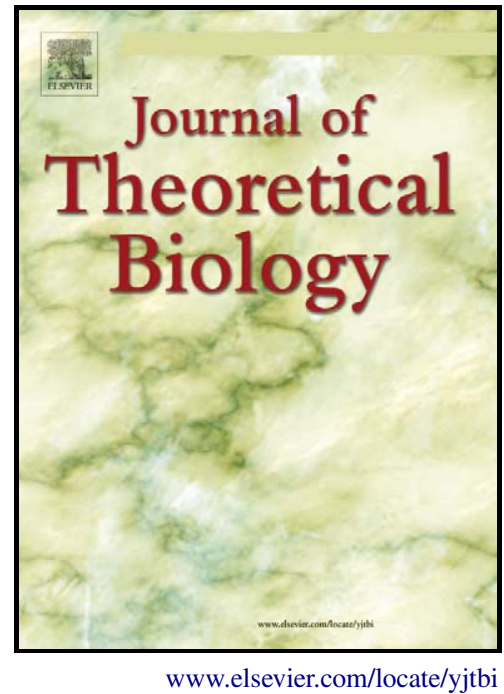

Received date: $\quad 7$ May 2009

Revised date: $\quad 25$ September 2009

Accepted date: $\quad 26$ September 2009

Cite this article as: Éva Kisdi, Costly dispersal can destabilize the homogeneous equilibrium of a metapopulation, Journal of Theoretical Biology, doi:10.1016/j.jtbi.2009.09.032

This is a PDF file of an unedited manuscript that has been accepted for publication. As a service to our customers we are providing this early version of the manuscript. The manuscript will undergo copyediting, typesetting, and review of the resulting galley proof before it is published in its final citable form. Please note that during the production process errors may be discovered which could affect the content, and all legal disclaimers that apply to the journal pertain. 


\title{
Costly dispersal can destabilize the homogeneous equilibrium of a metapopulation
}

\author{
Éva Kisdi \\ Department of Mathematics and Statistics \\ University of Helsinki, Finland
}

Running title: Costly dispersal can destabilize a metapopulation

Keywords: bifurcation, coupled map lattice, discrete-time population dynamics, dispersal, mortality, metapopulation, stability

Address for correspondence: Éva Kisdi, Department of Mathematics and Statistics, University of Helsinki, PO Box 68, FIN-00014 Finland. Phone: +358-9-1915 1489, fax: +358-9-1915 1400, email: eva.kisdi@helsinki.fi 


\begin{abstract}
I investigate the stability of the homogeneous equilibrium of a discrete-time metapopulation assuming costly dispersal with arbitrary (but fixed) spatial pattern of connectivity between the local populations. First, I link the stability of the metapopulation to the stability of a single isolated population by proving that the homogeneous metapopulation equilibrium, provided that it exists, is stable if and only if a single population, which is subject to extra mortality matching the average dispersal-induced mortality of the metapopulation, has a stable fixed point. Second, I demonstrate that extra mortality may destabilize the fixed point of a single population. Taken together, the two results imply that costly dispersal can destabilize the homogeneous equilibrium of a metapopulation. I illustrate this by simulations and discuss why earlier work, arriving at the opposite conclusion, was flawed.
\end{abstract}




\section{Introduction}

Discrete-time metapopulation models, cast in the form of coupled map lattices (CML) and related modelling frameworks, exhibit very rich dynamics even in the simplest case of a single species with passive (density-independent) dispersal. These riches include cyclic and chaotic behaviour with complicated bifurcation patterns, multiple attractors with fractal basins of attraction, and spatial pattern formation of various kinds (e.g. Kaneko 1990, 1998; Hastings 1993; Gyllenberg et al. 1993; Doebeli 1995; Lloyd 1995; Doebeli and Ruxton 1998; Utz et al. 2007). Because of this inherently complex dynamics, much work on discrete-time metapopulations falls back on numerical analysis and simulations, and hence is forced to commit to particular choices such as using the logistic map, or assuming nearest-neighbour dispersal or a Gaussian dispersal kernel. There are preciously few generalisations that hold true independently of the map chosen to describe the within-population dynamics and the pattern of coupling via dispersal between populations at various distances.

One such general result was given by Rohani et al. (1996) and, under more relaxed conditions, by Jang and Mitra (2000); both are special cases of the multi-species model of Jansen and Lloyd (2000). These authors proved that in a homogeneous metapopulation of a single species, where local populations are unstructured and interact solely via passive and cost-free dispersal, the metapopulation equilibrium where all local populations are characterised by the same equilibrium population density $\hat{N}$ is locally stable if and only if $\hat{N}$ is a stable equilibrium of a single population in absence of dispersal. Changes in cost-free passive dispersal thus do not affect the stability of the homogeneous equilibrium of a metapopulation. 
The appeal of this result is twofold. First, it holds for a large class of metapopulation models since it is independent of the form of local population dynamics and of the spatial pattern of dispersal (the fraction of individuals dispersing to various distances). Second, it establishes a simple link between the behaviour of an arbitrarily large metapopulation and the well-understood dynamics of a single isolated population. The result is however subject to the restrictive assumption that dispersal is free of any cost such as mortality during dispersal. In reality, dispersing individuals are often exposed to extra sources of mortality; passive dispersal in a fragmented habitat is especially likely to entail a high risk of death due to landing outside any suitable habitat fragment.

In this paper, I extend the analysis to costly dispersal, retaining arbitrary local dynamics and an arbitrary pattern of dispersal. I prove that the homogeneous equilibrium $(\hat{N}, \ldots, \hat{N})$ of a metapopulation is stable if and only if $\hat{N}$ is a stable equilibrium of an isolated population, which is subject to extra mortality that equals the average dispersal-related mortality of the metapopulation. This direct link between the dynamics of the metapopulation and the dynamics of an isolated population is an intuitive and straightforward extension of the result obtained by Rohani et al. (1996), Jang and Mitra (2000) and Jansen and Lloyd (2000), but it does not guarantee that the stability of the metapopulation is independent of dispersal. It is easy to construct examples of the single-population dynamics such that adding extra mortality destabilizes the equilibrium. With such dynamics within the local populations, increasing dispersal destabilizes the homogeneous equilibrium of the metapopulation. 
The effect of costly dispersal on metapopulation stability has been investigated by Ruxton et al. (1997a,b). My results contradict their conclusions: I explain in the Discussion why part of the results of Ruxton et al. (1997a,b) are not generalizable to arbitrary metapopulations and another part of their results is not correct.

\section{The model}

Consider a metapopulation of a single species, where all ecological interactions are local and populations are connected only by dispersal. Local populations are unstructured such that the within-population dynamics is given by

$$
N(t+1)=f(N(t))
$$

where $f: \mathbb{R}_{+} \rightarrow \mathbb{R}_{+}$may be arbitrary (e.g. may include an Allee-effect and may have several nontrivial equilibria, stable or not) but is the same in each population. $L$ local populations are connected via passive dispersal, such that a fixed fraction $m$ of individuals disperse, and a fraction $\varphi_{i j}$ of dispersers from population $j$ enter population

$i$. The matrix $\left(\varphi_{i j}\right)$ describes the pattern of dispersal. The dynamics of the metapopulation is thus given by

$$
N_{i}(t+1)=(1-m) f\left(N_{i}(t)\right)+m \sum_{j=1}^{L} \varphi_{i j} f\left(N_{j}(t)\right)
$$

for $i=1, \ldots, L$. At a homogeneous equilibrium $(\hat{N}, \ldots, \hat{N})$, 


$$
\hat{N}=\left[(1-m)+m \sum_{j=1}^{L} \varphi_{i j}\right] f(\hat{N})
$$

must hold for every index $i$. Hence for a homogeneous equilibrium to exist, one has to assume that $\sum_{j=1}^{L} \varphi_{i j}=s$ is the same for each destination patch $i$.

I make no assumption on the dispersal pattern $\left(\varphi_{i j}\right)$ other than $\sum_{j=1}^{L} \varphi_{i j}=s$ being the same for all $i$, i.e., that a homogeneous equilibrium exists. In particular, dispersal can have a mortality cost such that some of the emigrants die during dispersal and do not immigrate into any population. This implies that $\sum_{i=1}^{L} \varphi_{i j} \leq 1$ (with equality only for costfree dispersal); the sum $\sum_{i=1}^{L} \varphi_{i j}$ may in general be different for different source patches $j$. The mean probability of survival during dispersal (averaged over all source patches at the homogeneous equilibrium) is $s=\frac{1}{L} \sum_{j=1}^{L} \sum_{i=1}^{L} \varphi_{i j}$.

This general model subsumes, among others, the following cases of special interest:

(i) $L$ local populations (with arbitrary spatial locations) are connected via a global dispersal pool; each dispersing individual dies with probability $1-s$. In this particular case, $\varphi_{i j}=s / L$ for all pairs of patches $(i, j)$. 
(ii) $L$ populations form a 1-dimensional lattice with periodic boundaries, where dispersal depends on the distance between patches and dispersal mortality is also distance-related. In this case, $\varphi_{i j}$ is determined by $|i-j|$. More generally, $\varphi_{i j}$ may depend on the (signed) difference $i-j$, such that dispersal (and/or mortality) differs between left and right (e.g. there is a prevailing direction of wind). In these cases $\left(\varphi_{i j}\right)$ is a circulant matrix (cf. Rohani et al. 1996).

(iii) As in (ii), but the $L$ populations form a 2-dimensional lattice of size $L_{1} \times L_{2}$ with periodic boundaries; a vector of length $L=L_{1} L_{2}$ contains the local population densities $N_{i}$ of eqs. (2). In this case, $\left(\varphi_{i j}\right)$ is a "circulant of circulants", i.e., a block circulant where each block itself is a circulant. (Rohani et al. (1996) assumed erroneously that $\left(\varphi_{i j}\right)$ itself is a circulant also for a 2dimensional lattice: this is not the case, see e.g. Fig. 1 of White and White (2005).)

\section{Stability of the homogeneous equilibrium}

Consider an isolated population

$$
N(t+1)=\alpha f(N(t))
$$

with $0<\alpha \leq 1$. From eq. (3) it is obvious that with $\alpha=1-m+m s$, the equilibrium density of this isolated population, $\hat{N}$, equals the local densities of a metapopulation at the homogeneous equilibrium, $(\hat{N}, \ldots, \hat{N})$. In the metapopulation context, $\alpha$ is the 
probability of not dying due to dispersal (either not dispersing or surviving dispersal). The behaviour of the metapopulation is linked to the local dynamics by the following proposition:

The homogeneous equilibrium $(\hat{N}, \ldots, \hat{N})$ of the metapopulation model in eq. (2), provided that it exists, is locally asymptotically stable if and only if the fixed point $\hat{N}$ of the single-population dynamics in eq. (4), with extra mortality matching the average dispersal-induced mortality at the homogeneous metapopulation equilibrium [ $\alpha=1-m+m s]$, is locally asymptotically stable.

To prove this claim, perform a standard stability analysis by substituting $N_{i}(t)=\hat{N}+n_{i}(t)$ into eq. (2). Linearization yields $\mathbf{n}(t+1)=f^{\prime}(\hat{N}) \mathbf{B n}(t)$, where matrix B has the elements

$$
b_{i j}=\left\{\begin{array}{cc}
1-m+m \varphi_{i i} & \text { for } i=j \\
m \varphi_{i j} & \text { for } i \neq j
\end{array}\right.
$$

and, by the assumption that the homogeneous equilibrium exists,

$$
\sum_{j=1}^{L} b_{i j}=1-m+m \sum_{j=1}^{L} \varphi_{i j}=1-m+m s=\alpha \text { for all } i
$$

$\frac{1}{\alpha} \mathbf{B}$ is therefore a stochastic matrix (all elements are non-negative and each row sums to 1$)$. Since the leading eigenvalue of a stochastic matrix is 1 , the leading eigenvalue of B must be $\alpha$; and the eigenvalue of the Jacobian matrix $f^{\prime}(\hat{N}) \mathbf{B}$ with greatest 
modulus is $\alpha f^{\prime}(\hat{N})$. Hence $(\hat{N}, \ldots, \hat{N})$ is stable if $\left|\alpha f^{\prime}(\hat{N})\right|<1$, which is precisely the same as the condition for the single-population dynamics in eq. (4) having a stable equilibrium at $\hat{N}$.

This proof is a straightforward extension of Jang and Mitra (2000) to the case of costly dispersal (but as Silva et al. (2001) also noticed, matrix $\left(\varphi_{i j}\right)$ need not be irreducible as Jang and Mitra (2000) required for the proof to hold; indeed, two unconnected networks of populations with identical within-population dynamics and identical value of $\alpha$ yield a reducible matrix for $\left(\varphi_{i j}\right)$, yet the two networks will independently equilibrate to the same homogeneous equilibrium upon a small perturbation). The same result can also be obtained from the framework of Jansen and Lloyd (2000) by introducing some cost to dispersal (but their technical assumption that $\left(\varphi_{i j}\right)$ is diagonalizable is not necessary for the proof above). The pattern of dispersal need not be shift invariant, i.e., $\left(\varphi_{i j}\right)$ need not be circulant as assumed by Rohani et al. (1996).

If dispersal entails no cost ( $\alpha=1$ ), then the homogeneous equilibrium of the metapopulation is stable whenever $\left|f^{\prime}(\hat{N})\right|<1$, i.e., whenever a single population has a stable equilibrium, as found earlier. Costly dispersal destabilizes the metapopulation under precisely the same conditions as when extra mortality destabilizes a single population. I shall thus explore when decreasing $\alpha$ in eq. 4 leads to loss of stability. 


\section{Destabilization by extra mortality}

A fixed point $\hat{N}$ of the single-population model in eq. (4) undergoes a bifurcation when $\left|\alpha f^{\prime}(\hat{N})\right|=1$. Here I shall focus on the case when $\alpha f^{\prime}(\hat{N})=-1$ and extra mortality (somewhat counter-intuitively) increases the propensity for "boom and bust" behaviour, such that population cycles replace a stable equilibrium. In the alternative case of $\alpha f^{\prime}(\hat{N})=1$ (with $\hat{N}>0$ ), the fixed point generically undergoes a tangent bifurcation and disappears. Extra mortality can then drive the population extinct or can send it to some other attractor, and destabilization can occur because the population may be attracted to a limit cycle or to chaos when $\hat{N}$ is lost. What happens, however, depends on the concrete model at hand and cannot be predicted from the local properties of $\hat{N}$.

Suppose that the parameters of $f$ are chosen such that $\alpha f^{\prime}(\hat{N})=-1$ occurs at $\alpha=\alpha_{0}$; for example, one can set $\alpha_{0}=1$ by choosing the parameters of $f$ such that the dynamics is at a period-doubling bifurcation point in absence of any extra mortality. Implicit differentiation of the equilibrium condition $\alpha f(\hat{N})-\hat{N}=0$ yields $[d \hat{N} / d \alpha]_{\alpha=\alpha_{0}}=f(\hat{N}) /\left[1-\alpha_{0} f^{\prime}(\hat{N})\right]=\hat{N} / 2$, and therefore

$\left.\frac{d\left[\alpha f^{\prime}(\hat{N})\right]}{d \alpha}\right|_{\alpha=\alpha_{0}}=f^{\prime}(\hat{N})+\left.\alpha_{0} f^{\prime \prime}(\hat{N}) \frac{d \hat{N}}{d \alpha}\right|_{\alpha=\alpha_{0}}=-\frac{1}{\alpha_{0}}+\frac{1}{2} \alpha_{0} f^{\prime \prime}(\hat{N}) \hat{N}$ 
Adding extra mortality (decreasing $\alpha$ ) will destabilize the fixed point (decrease the value of $\alpha f^{\prime}(\hat{N})$ beyond -1) if the right hand side of eq. (7) is positive, i.e., if $f$ is sufficiently convex such that

$$
f^{\prime \prime}(\hat{N})>\frac{2}{\alpha_{0}^{2} \hat{N}}
$$

holds at the bifurcation point $\alpha_{0} f^{\prime}(\hat{N})=-1$. In the opposite case, adding extra mortality stabilizes the fixed point.

It is straightforward to construct biologically plausible maps $f$ for which condition (8) is satisfied and therefore extra mortality has a destabilizing effect. To do this, simply choose $\alpha_{0}$ (where the bifurcation is to occur) and $\hat{N}$ (which can be scaled to 1 without loss of generality). Then choose $f$ to satisfy the local conditions $\alpha_{0} f(\hat{N})=\hat{N}$, $\alpha_{0} f^{\prime}(\hat{N})=-1$ and $f^{\prime \prime}(\hat{N})$ sufficiently large positive such that condition (8) holds; the rest of the function can be chosen arbitrarily and such that it is biologically interpretable [e.g. $f(0)=0$ ]. An example is shown in Fig. 1a. Increasing extra mortality by decreasing $\alpha$ shifts the fixed point to the left, and makes the function $\alpha f(N)$ less steep at every point: Hence to destabilize the fixed point, $f$ must be sufficiently convex such that the shift towards the left makes the slope steeper (more negative) at the fixed point, despite that at every individual point the function is becoming less steep [this verbal condition is quantified by (8)]. The example in Fig. 1 was chosen to make this visually clear; but the function does not have to be so exaggerated as this example. Note that the shape of $f$ right to the fixed point with $\alpha=1$ 
( $N>1.15$ in Fig. 1a) is irrelevant for the stability of the fixed point with any extra mortality $(\alpha \leq 1)$.

Fig. 1b shows the bifurcation diagram of the single-population dynamics in eq. (4) with the map $\alpha f$ as in Fig. 1a. The population is viable for $\alpha>0.24$; the nontrivial fixed point is unstable and the population has an attracting 2-cycle for $0.5<\alpha<0.78$. There is another attracting 2-cycle that appears at $\alpha=0.9$ via a tangent bifurcation of the second iterated map $\alpha f(\alpha f(N))$ and undergoes a rapid period-doubling cascade, but this does not affect the stability of the fixed point of $\alpha f(N)$.

\section{Destabilization by costly dispersal}

As proven in section 3, the homogeneous equilibrium of a metapopulation is stable if and only if the fixed point of the single-population dynamics with $\alpha=1-m+m s$ is stable, where $m$ is the fraction of individuals who disperse and $s$ is the average survival of dispersers. If the single-population fixed point is unstable in the interval $\left(\alpha_{1}, \alpha_{0}\right)$, then the homogeneous metapopulation will be unstable, irrespectively of the pattern of dispersal, for the dispersal fractions $\left(1-\alpha_{0}\right) /(1-s)<m<\left(1-\alpha_{1}\right) /(1-s)$.

As an example, consider a metapopulation like in Ruxton et al. (1997), where 10 patches are arranged in a circle and dispersers are evenly divided between the two nearest neighbour patches. Assume that local population dynamics is given by the map $f$ shown in Fig. 1a (thick line). As Fig. 2 illustrates, the homogeneous equilibrium is stable in absence of dispersal, but costly dispersal destabilizes the equilibrium for some values of $m$. 


\section{Discussion}

In this paper, I obtained two results: (1) The homogeneous equilibrium of a metapopulation, provided that it exists, is stable if and only if a single population, which is subject to extra mortality matching the average dispersal-induced mortality of the metapopulation, has a stable fixed point; (2) Extra mortality may destabilize the fixed point of a single population. Taken together, these imply that costly dispersal can destabilize the homogeneous equilibrium of a metapopulation (see an example in Fig. 2). Costly dispersal affects metapopulation stability exclusively due to the extra mortality it implies: If extra mortality stabilizes the fixed point of an isolated population then costly dispersal stabilizes the homogeneous equilibrium of a metapopulation, and if extra mortality destabilizes an isolated population then costly dispersal destabilizes the metapopulation. Cost-free dispersal has no effect (cf. Rohani et al. 1996; Jang and Mitra 2000; Jansen and Lloyd 2000). Because the effect on stability is independent of any aspect of dispersal but the average cost, costly dispersal can destabilize the homogeneous equilibrium even in the simplest metapopulation model with a global dispersal pool.

Ruxton et al. (1997a,b) have previously investigated the effect of costly dispersal on the dynamics of a metapopulation, and they concluded that costly dispersal stabilizes the homogeneous equilibrium. In particular, they found that extra mortality has a stabilising effect in isolated populations, and that the stabilizing effect of costly dispersal in a metapopulation is even stronger than the stabilizing effect of extra mortality in an isolated population. My results contradict these conclusions. First, I 
have proven that there is no difference between the stabilizing effect of extra mortality and the stabilizing effect of costly dispersal; costly dispersal affects stability only via the extra mortality. This discrepancy is due to a simple algebraic error in the proof of Ruxton et al. (1997b). [In equations (B.2) and (B.3) of Ruxton et al. (1997b), the derivative of the within-population map should appear without being multiplied by $1-\mu \delta$, the probability of not dying due to dispersal in their notation; but their $\mathrm{Q}$ contains this extra multiplicative factor, so that they account for dispersal-related mortality twice. The same results are reproduced as equations (5) and (6) of Ruxton et al. (1997a).] Second, Ruxton et al. (1997a,b) found that extra mortality stabilizes the fixed point in an isolated population, but their analysis assumed a particular form of local population dynamics. Extra mortality indeed has a stabilizing effect in the model they studied and also in other widely used discrete-time population models, but this is not true in general for all biologically plausible dynamics.

Adding extra mortality destabilizes the fixed point of a single-population model if $f^{\prime \prime}(\hat{N})>2 / \hat{N}$ holds at the bifurcation point $f^{\prime}(\hat{N})=-1$ (this is inequality (8) with $\alpha_{0}$ set to 1 by factoring it into $f$ ), i.e., if the map $f$ is sufficiently convex at the point of period-doubling bifurcation. All overcompensating models with a differentiable and positivity-preserving map $f$ have convex parts (the concave quadratic or logistic map needs to be truncated to avoid negative population densities). The few widely used maps, however, fail to satisfy condition (8), because the bifurcation does not occur in the convex part or the function is not convex enough. Consider the Ricker map, $f(N)=\lambda N e^{-a N}$, where $\alpha_{0}$ is factored into $\lambda$. The Ricker model has a single nontrivial fixed point at $\hat{N}=(\ln \lambda) / a$ and undergoes a period-doubling bifurcation at 
$\lambda=e^{2}$. At the bifurcation point, therefore, $\hat{N}=2 / a$ and $f^{\prime \prime}(\hat{N})=0$; i.e., the bifurcation happens exactly when the fixed point passes through the point of inflection of $f$. Condition (8) is never satisfied in the Hassell (1975) model $\left[f(N)=\lambda N /(1+a N)^{b}\right]$ or in the Maynard Smith and Slatkin (1973) model $\left[f(N)=\lambda N /\left(1+(a N)^{b}\right)\right]$ either; the latter was used by Ruxton et al. $(1997 \mathrm{a}, \mathrm{b})$.

This of course does not mean that extra mortality could not destabilize the fixed point with other maps, and indeed it is easy to construct biologically reasonable maps where extra mortality is destabilizing. Fig. 1 shows an example where the relevant property of $f$ is visually clear: multiplying $f$ with $\alpha<1$ makes the slope steeper at the fixed point. But $f$ does not have to be as strongly convex as in Fig. 1a. A small "dent" added on the Maynard Smith and Slatkin (1973) model, for example, is enough to satisfy inequality (8) and to destabilize the fixed point under extra mortality. We know relatively little about the underlying mechanisms that create the discrete-time maps (see e.g. Gyllenberg et al. 1997; Gamarra and Sole 2002; Johansson and Sumpter 2003; Thieme 2003; Geritz and Kisdi 2004; Eskola and Geritz 2007; Eskola and Parvinen 2007 for mechanistic underpinnings of various discrete-time models), and therefore we don't have a priori constraints on the convexity of $f$. Empirical data are usually too noisy to ascertain the precise shape of $f$. There is thus no ground for narrowing research to the few famous discrete-time population models. As the present study also underlines, it is dangerous to overuse just a few models, because results based on them may not carry over to other models.

To put the present results into proper perspective, note that a metapopulation may have alternative attractors next to its homogeneous equilibrium. For example, equilibria 
with unequal population densities exist when an Allee-effect operates within local populations and dispersal is weak (see Gruntfest et al. 1997; Amarasekare 1998; Gyllenberg et al. 1999). Cyclic or chaotic attractors may also coexist with the homogeneous equilibrium; in fact, an asynchronous (possibly chaotic) attractor exists in the example of Fig. 2 for some small values of $m$ (not shown), where the homogeneous equilibrium is also stable. The existence and stability of these alternative attractors generally depend on dispersal even if dispersal is passive and cost-free.

As shown here, costly passive dispersal can either destabilize or stabilize the homogeneous equilibrium, depending on the properties of local population dynamics (see condition (8)). It is well known that destabilization can occur also in other ways. The homogeneous equilibrium can be destabilized if dispersal is cost-free but depends on local population density (Ruxton 1996; Jang and Mitra 2000; Silva et al. 2001). Destabilization can result also if the local population dynamics has more than one variable, either because populations are structured e.g. by age or because there are several interacting species (such as predator-prey or host-parasitoid), provided that different age/stage classes or different species differ in dispersal (Hastings 1992; Rohani and Ruxton 1999a,b; Jansen and Lloyd 2000; White and White, 2005; de Castro et al. 2006). Concerning the reverse case, cost-free dispersal cannot stabilize the homogeneous equilibrium if it is unstable in absence of dispersal: this holds for density-dependent dispersal (Silva et al. 2001) and for multi-species systems (Jansen and Lloyd 2000) as well. A simple continuity argument shows that adding dispersal cost can change this conclusion. If dispersal cost is stabilizing (as it is the case for several widely used models of within-population dynamics, see above) whereas density dependence of dispersal is weak and different species have sufficiently similar 
dispersal behaviour, then dispersal will have an overall stabilizing effect on the homogeneous equilibrium of the metapopulation. Because dispersal costs are ubiquitous in nature and they may stabilize as well as destabilize the metapopulation, general inferences on the effect of dispersal seem unfortunately not possible.

\section{Acknowledgements}

This research was financially supported by the Academy of Finland. 


\section{References}

Amarasekare P. 1998. Interactions between local dynamics and dispersal: Insights from single-species models. Theor. Pop. Biol. 53:44-59.

de Castro M. L., J. A. L. Silva \& D. A. R. Justo. 2006. Stability in an age-structured metapopulation model. J. Math. Biol. 52:183-208.

Doebeli M. 1995. Dispersal and dynamics. Theor. Pop. Biol. 47:82-106.

Doebeli M. \& G. D. Ruxton. 1998. Stabilization through spatial pattern formation in metapopulations with long-range dispersal. Proc. R. Soc. Lond. B 265:1325-1332.

Eskola H. \& S. A. H. Geritz. 2007. On the derivation of discrete-time population models by varying within-season patterns of reproduction and aggression. Bull. Math. Biol. 69: 329-346.

Eskola H. T. M. \& K. Parvinen. 2007. On the mechanistic underpinning of discretetime population models with Allee effect. Theor. Pop. Biol. 72: 41-51.

Gamarra J. G. P. \& R. V. Sole. 2002. Complex discrete dynamics from simple continuous population models. Bull. Math. Biol. 64:611-620. 
Geritz S. A. H. \& E. Kisdi. 2004. On the mechanistic underpinning of discrete-time population models with complex dynamics. J. theor. Biol. 228:261-269.

Gruntfest Y., R. Arditi \& Y. Dombrovsky. 1997. A fragmented population in a varying environment. J. theor. Biol. 185:539-547.

Gyllenberg M., G. Söderbacka \& S. Ericsson. 1993. Does migration stabilize local population dynamics? Analysis of a discrete metapopulation model. Math. Biosci. $118: 25-49$.

Gyllenberg M., I. Hanski \& T. Lindström. 1997. Continuous versus discrete single species population models with adjustable reproductive strategies. Bull. Math. Biol. 59:679-705.

Gyllenberg M., J. Hemminki \& T. Tammaru. 1999. Allee effects can both conserve and create spatial heterogeneity in population densities. Theor. Pop. Biol. 56:231-242.

Hassell M. P. 1975. Density-dependence in single-species populations. J. Anim. Ecol. $44: 283-295$.

Hastings A. 1992. Age dependent dispersal in not a simple process: Density dependence, stability, and chaos. Theor. Pop. Biol. 41:388-400.

Hastings A. 1993. Complex interactions between dispersal and dynamics: Lessons from coupled logistic equations. Ecology 74:1362-1372. 
Jang S. R.-J. \& A. K. Mitra. 2000. Equilibrium stability of single-species metapopulations. Bull. Math. Biol. 62: 155-161.

Jansen V. A. A. \& A. L. Lloyd. 2000. Local stability analysis of spatially homogeneous solutions of multi-patch systems. J. Math. Biol. 41: 232-252.

Johansson A. \& D. J. T. Sumpter. 2003. From local interactions to population dynamics in site-based models of ecology. Theor. Pop. Biol. 64:497-517.

Kaneko K. 1990. Supertransients, spatiotemporal intermittency and stability of fully developed spatiotemporal chaos. Physics Letters A 149: 105-112.

Kaneko K. 1998. Diversity, stability, and metadynamics: Remarks from coupled map studies. In: J. Bascompte \& R. V. Sole, (eds): Modeling spatiotemporal dynamics in ecology. Springer, pp. 27-45.

Lloyd A.L. 1995. The coupled logistic map: A simple model for the effects of spatial heterogeneity on population dynamics. J. theor. Biol. 173:217-230.

Maynard Smith J. \& M. Slatkin. 1973. The stability of predator-prey systems. Ecology $54: 384-391$.

Rohani P., R. M. May \& M. P. Hassell. 1996. Metapopulations and equilibrium stability: The effects of spatial structure. J. theor. Biol. 181:97-109. 
Rohani P. \& G. D. Ruxton. 1999a. Dispersal and stability in metapopulations. IMA Journal of Mathematics Applied in Medicine and Biology 16: 297-306.

Rohani P. \& G. D. Ruxton. 1999b. Dispersal-induced instabilities in host-parasitoid metapopulations. Theor. Pop. Biol. 55: 23-36.

Ruxton G. D. 1996. Density-dependent migration and stability in a system of linked populations. Bull. Math. Biol. 58: 643-660.

Ruxton G., J. L. Gonzalez-Andujar \& J. N. Perry. 1997a. Mortality during dispersal stabilizes local population fluctuations. J. Anim. Ecol. 66: 289-292.

Ruxton G. D., J. L. Gonzalez-Andujar \& J. N. Perry. 1997b. Mortality during dispersal and the stability of a metapopulation. J. theor. Biol. 186: 389-396.

Silva J. A. L., M. L. de Castro \& D. A. R. Justo. 2001. Stability in a metapopulation model with density-dependent dispersal. Bull. Math. Biol. 63:485-505.

Thieme H. R. 2003. Mathematics in population biology. Princeton University Press, Princeton.

Utz M., E. Kisdi \& M. Doebeli. 2007. Quasi-local competition in stage-structured metapopulations: A new mechanism of pattern formation. Bull. Math. Biol. 69: 16491672. 
White S. M. \& K. A. J. White. 2005. Relating coupled map lattices to integrodifference equations: dispersal-driven instabilities in coupled map lattices. J. theor. Biol. 235: 463-475. 


\section{Figure legends}

Figure 1. Destabilization by extra mortality. (a) An example for the map $\alpha f(N)$ in the single-population dynamics in eq. (4) such that extra mortality destabilizes the fixed point. Thick line: the map without extra mortality ( $\alpha=1$, the fixed point is stable); dashed line: the map with extra mortality ( $\alpha=0.7$, the fixed point is unstable). $f$ was obtained as an Interpolation function of Mathematica ${ }^{\circledR}$ with points and derivatives as follows: $(N, f)=(0,0)$ with derivatives $f^{\prime}(0)=4.25$ and $f^{\prime \prime}(1)=-4.25$;

$(N, f)=(1,1.275)$ with derivatives $f^{\prime}(1)=-1.275, f^{\prime \prime}(1)=6.8$ and $f^{\prime \prime \prime}(1)=-12.75$; $(N, f)=(2,0)$ with slope $f^{\prime}(1)=-1.36$. (b) Bifurcation diagram of the singlepopulation model with $\alpha f$ as in (a).

Figure 2. Simulated dynamics of a 10-patch metapopulation with periodic boundaries and nearest-neighbour dispersal with $s=0.2$; local population dynamics is given by the map in Fig. 1a (thick line). Since the single-population fixed point is unstable for $0.5<\alpha<0.78$, the homogeneous equilibrium of the metapopulation must be unstable for dispersal fractions $0.275<m<0.625$ (see text). Starting from the vicinity of the homogeneous equilibrium (with an independent small perturbation added to each population), 5000 generations were simulated to let the transients die out and the subsequent 50 generations are shown. (a) $m=0$, the homogeneous equilibrium is stable in absence of dispersal; (b) $m=0.5$, the homogeneous equilibrium is unstable with costly dispersal and the metapopulation has converged to a synchronous 2-cycle. 
(a)

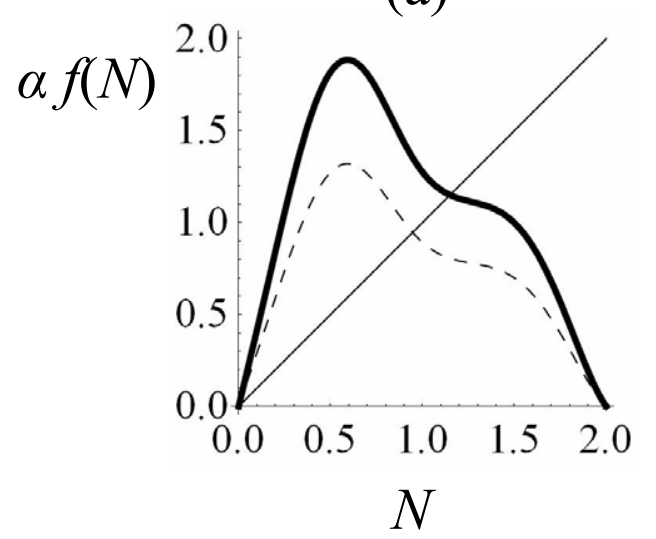

(b)

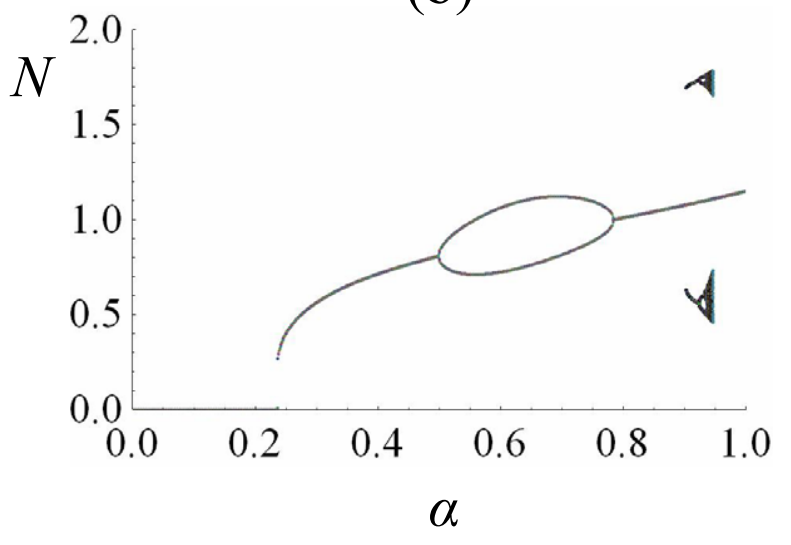

Figure 1 

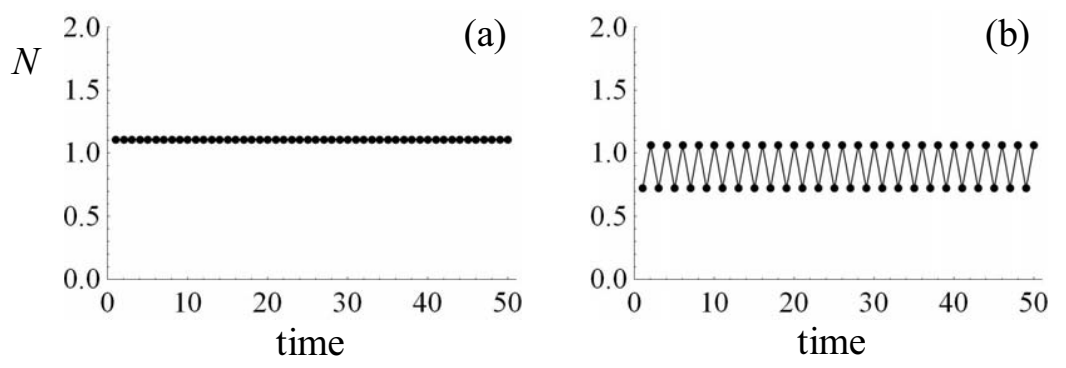

Figure 2 